uphold. We currently capture patient feedback in a variety of ways which has adequately fed back patient views. However, we needed a tool to demonstrate the complexity of the services that we offer in response to patient needs; one that would also enable us to reflect on our services and instigate change if needed.

Methods After thorough investigations into patient feedback methods, we decided to use patient case studies. A pro-forma was developed, giving a rationale for their use, notes for guidance, and a template for staff to complete. The template also contained a tool at the bottom auditing the case study against CQC lines of enquiry. This was included so the organisation is able to monitor that services are achieving, and are responsive to, all key lines of inquiry from the CQC. We decided to get one patient case study each month, on a rolling programme of services we provide.

Results and Conclusion Patient case studies have enabled the organisation to respond to complex patient needs, consistently creating services that are directly patient-led in their design and focus. A great example of this was a patient case study that highlighted how difficult it is to have an end of life discussion with a patient's relatives when they speak a different language and the patient is the only person able to interpret. This led to the organisation contracting a language line service which will now be utilised in these situations, leading to an improved patient experience.

\section{P-136 HOSPICE IN-PATIENT UNIT: IMPROVING FALLS MANAGEMENT}

Rosie Ingham, Jimmy Brash, Andrew Fletcher, Tracy Parkinson, Deborah Seed, Barbara Sutton. St Catherine's Hospice, Lancashire, UK

\subsection{6/bmjspcare-2017-hospice. 161}

Background All patients on the in-patient unit (IPU) are potentially at risk of falling (Hospice UK 2016). Care of a patient post fall, who required hospitalisation had led to a review of falls documentation and processes. As part of a wider IPU project reviewing ways of working, falls was identified as an ongoing priority.

Aims 1. To improve patient care and safety

2. Improve quality of documentation in line with national guidance

3. Meet requirements for regulatory bodies

4. Reduce number of falls.

Method A retrospective audit of incident reports was conducted, identifying documentation was not always completed correctly. Staff feedback showed existing falls assessments were confusing, leading to inconsistent completion. A staff nurse with an existing interest in falls management was identified to lead the work stream and a work plan formulated. This included:

- Review of national guidance and liaison with local falls teams

- Review hospice risk assessments and care plans pre-andpost fall

- Update hospice policy

- Documentation to be integrated into the electronic patient record.

Results A new falls risk screening tool, falls diary and care plan have been implemented following review of local and national guidelines and have been integrated with the electronic patient record. Staff training has been provided, increasing staff awareness regarding all aspects of falls management.

The assessment of patients and families approach to risk of falls has led to a falls prevention leaflet which will be given to patients and families on admission. This work stream has also prompted review of falls prevention equipment and led to purchasing additional bed sensors and bedside tidy boxes.

Conclusion Completed work to date has introduced new documentation and increased staff's knowledge and skills in relation to falls prevention. Over the next few months an audit of the new documentation will be conducted to evaluate effectiveness.

\section{P-137 PHYSIOTHERAPY PALLIATIVE CANCER CARE: LEARNING THROUGH INNOVATION}

${ }^{1,2}$ Caroline Belchamber, ${ }^{2}$ Elizabeth Rosser, ${ }^{2}$ Caroline Ellis-Hill. 'Sue Ryder, London, UK; ${ }^{2}$ Bournemouth University, Bournemouth, UK

\subsection{6/bmjspcare-2017-hospice. 162}

Background The end-of-life framework to improve care efficiency has fuelled the desire to achieve high-quality care, where innovation and evaluation are central components of change. However, implementation of innovations remains challenging, with a lack of understanding regarding the role and attributes of policy champions in the innovation process.

Aim The aim of this study was to explore a local palliative cancer care physiotherapy service provision in the light of best practice recommendations.

Method A single qualitative case study design provided a framework to facilitate the inclusion of multiple perspectives in a complex context within an area of healthcare where little research has been undertaken. Multiple methods of data collection gained a variety of perspectives, which included four data sets: interviews (healthcare professionals [10]); interviews (service users [10]); observations (physiotherapists [2] treating service users [5]); and policy document collection (NICE guidelines; white papers [12]). This was combined with a practice development project, which used a concept analysis approach and improvement framework to enable best practice to be implemented. A thematic approach to data analysis was used within and across the four datasets.

Findings The research and practice development project combined to evidence the metamorphosis of both the physiotherapist profession and service provision where competencies around mentorship, entrepreneurship, leadership, policy championship, integrated teamwork, humanisation and self-reflection embodied within the psycho-social-cultural-spiritual model of healthcare enabled them to meet key policy recommendations of service quality and innovation.

Conclusion Physiotherapists responded to the changing palliative cancer care environment, through the metamorphosis of their service provision and by addressing two out of the three key policy recommendations i) service quality and ii) innovation but not iii) measurement of service quality and innovation. A debate is necessary around the need for profession specific or service specific outcomes in this area and how physiotherapists can 'prove their worth.' 